\title{
PRESENCE D'ONCHOCERCA TARSICOLA Bain et Schulz-Key, 1974 CHEZ LE RENNE (1)
}

\author{
par O. Bain (*), C. Rehbinder (*) et G. Petit (*) \\ (*) Laboratoire de Zoologie (Vers), associé au C.N.R.S., Muséum national d'Histoire naturelle, \\ 43, rue Cuvier, F 75231 Paris Cedex 05. \\ (*) Sw. Univ. of Agricult. Sciences, College of Vet. Medicine, \\ Department of Pathology, S-750 07 Uppsala. Sweden.
}

\section{Résumé.}

O. tarsicola, décrit initialement chez Cervus elaphus, est retrouvé chez Rangifer tarandus, en Suède. Trois faits indiquent que c'est le Cerf qui paraît être l'hôte fondamental :

1. Le Renne est parasité seulement par $O$. tarsicola tandis que des spéciations se sont effectuées chez le Cerf (trois espèces de la même lignée).

2. O. tarsicola, fondamentalement logé au niveau des tendons des pattes, présente en outre des localisations aberrantes chez le Renne: ce phénomène est souvent observé chez les parasites transfuges.

3. Les fortes infestations à $O$. tarsicola chez le Renne semblent dater de la fin des années 60 et, depuis, elles s'étendent de plus en plus.

\section{Summary.}

Occurence of Onchocerca tarsicola Bain and Schulz-Key, 1974, in reindeer.

Onchocerca tarsicola is recorded from reindeer (Rangifer tarandus L.). This parasite is originally described from red deer (Cervus elaphus L.). There are indications that reindeer may not be the primary host of O. tarsicola:

1. Reindeer is parasitized only by $O$. tarsicola whereas red deer, due to speciations, is carrying three related Onchocerca species from the same line.

2. O. tarsicola, usually found along tendons distal of the tarsal and carpal joints, is in reindeer frequently found at aberrant locations which is commonly observed in connection with " transfuga" parasites.

3. Heavy infestations of O. tarsicola in reindeer seem to have started in the late sixties and since then have become more and more extensive.

(1) Travail effectué grâce à une subvention de 1'Organisation Mondiale de la Santé.

Accepté le 30 mars 1979. 
Des Onchocerques non identifiées ont été signalées à plusieurs reprises chez le Renne, Rangifer tarandus, au niveau des ligaments et des tendons des pattes, dans des petits nodules sous-cutanés ventraux et sous le péritoine (cf. Rehbinder, 1973).

Des prélèvements de tissus parasités ont été effectués en 1977 et 1978 à Angesa, au cours de la saison d'abattage des Rennes (août-septembre).

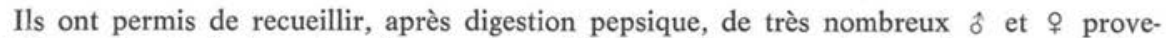
nant des pattes, $1 \delta^{\hbar}$ et un long fragment postérieur de $q$ provenant de nodules sous-péritonéaux, $2 \delta, 1 q$ et des fragments de $q$ provenant de nodules sous-cutanés ventraux.

Toutes ces Onchocerques sont identifiables à O. tarsicola Bain et Schulz-Key, 1974, initialement décrite chez le Cerf, Cervus elaphus, en Allemagne et très caractéristique par sa grande et très épaisse microfilaire $(400 \mu \mathrm{m} / 11-12 \mu \mathrm{m})$. Nous figurons la vue ventrale de la queue d' $1 \hat{\delta}$, non pas parce qu'elle est différente des spécimens du Cerf, mais parce qu'un matériel plus abondant a permis de préciser la place des phasmides (fig. 1).

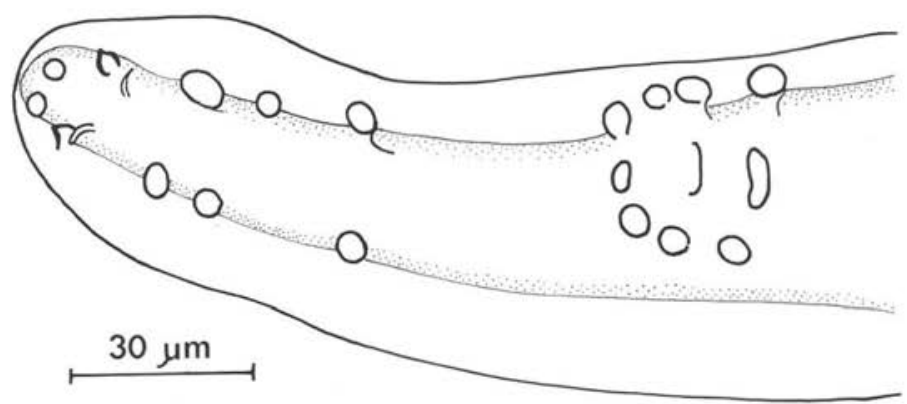

Fig. 1, O. tarsicola Bain et Schulz-Key, 1974 chez le Renne; queue d'un $\delta^{\prime}$, vue ventrale (les phasmides sont à la base de la $9^{\mathrm{c}}$ paire de papilles; celle-ci est transformée en pointes fortement cuticularisées).

La question qui se pose est de savoir quel est l'hôte fondamental de cette Onchocerque.

Trois faits nous font penser que c'est le Cerf :

1. - Les phénomènes de spéciation d'un parasite à l'intérieur d'un même hôte sont un processus extrêmement fréquent en parasitologie; ils impliquent une très longue durée d'évolution chez l'hôte et aboutissent chez celui-ci à la coexistence de plusieurs espèces congénères (Chabaud et Durette-Desset, 1979).

Or, une seule Onchocerque, O. tarsicola, semble parasiter le Renne, tandis que, chez le Cerf, une diversification s'est effectuée et 3 espèces proches coexistent, $O$. tarsicola, O. tubingensis Bain et Schulz-Key, 1974, et O. garmsi Bain et Schulz-Key, 1976 (2).

(2) La $4^{e}$ Onchocerque, O. flexuosa (Wedl, 1856), appartient à une lignée évolutive différente. 
2. - Les localisations des Onchocerques chez le Cerf ont fait l'objet de longues et précises recherches (Schulz-Key, 1975), et O. tarsicola a été trouvé seulement au niveau des articulations tibiotarsales et radiocarpales; au contraire, chez le Renne, les localisations aberrantes (nodules sous-cutanés ventraux et sous-péritonéaux) ne sont pas rares, et celles-ci, en règle générale, s'observent chez les parasites transfuges et non chez les parasites infestant leur hôte d'origine.

3. - Les infections à $O$. tarsicola chez le Renne ont été signalées pour la première fois vers la fin des années 60 et sont, depuis, devenues de plus en plus importantes (Rehbinder et al., 1976), ce qui semble indiquer une introduction récente du parasite.

\section{Bibliographie}

Bain O., Schulz-Key H. (1974) : Les Onchocerques du Cerf européen : redescription d'O. flexuosa (Wedl, 1856) et description d'O. tubingensis n. sp. et O. tarsicola n. sp., Tropenmel. Parasit., 25, 437-449.

Bain O., Schulz-Key H. (1976) : Une quatrième espèce d'Onchocerque, O. garmsi n. sp. chez le Cerf européen. Tropenmed. Parasit., 27, 474-478.

Chabaud A.-G., Durette-Desset M.-C. (1979) : Parasitisme par plusieurs espèces congénériques. Bull. Soc. Zool. France (sous presse).

Rehbinder C. (1973): Note on Onchocercosis in Swedish Reindeer (Rangifer tarandus). Acta Vet. Scand., 14, 642-644.

Rehbinder C., Christensson D., Glatthard V. (1976). Parasitic granulomas in Swedish forest reindeer (Rangifer tarandus). In Wildlife Diseases. Ed. L. A. Page, Plenum Press, édit., New-York, pp. $597-$ 607.

Schulz-Key H. (1975) : Untersuchungen über die Filarien der Cervidien in Süd-deutschland 2. Die Filarien des Rothirsches (Cervus elaphus). Tropenmed. Parasit., 26, 348-358. 